\title{
Reducing Driver Task Load and Promoting Sociability through an Affective Intelligent Driving Agent (AIDA)
}

\author{
Kenton Williams and Cynthia Breazeal \\ Massachusetts Institute of Technology, MIT Media Lab, 20 Ames St., E15-468, \\ Cambridge, MA, United States of America \\ \{kjwillia, cynthiab\} @media.mit.edu
}

\begin{abstract}
This work outlines the development of an Affective Intelligent Driving Agent (AIDA), a social robot that sits in a vehicle's dashboard and behaves as a friendly assistant. This highly expressive robot uses an Android smartphone as its face, which serves as the main computational unit for the system. AIDA determines what information may be relevant to the driver, delivers it at the most appropriate time, and resolves which expressions should be used when doing so. An evaluation was performed in which participants completed mock driving tasks with the aid of 1) a smartphone with apps, 2) AIDA as a static, expressive agent, or 3) AIDA as a mobile robot. Results showed that the AIDA robot helped reduce user task load and promoted more sociability with users better than the smartphone or AIDA as a static agent.
\end{abstract}

Keywords: Affective HCI, Human-robot interaction, Novel user interfaces and interaction techniques.

\section{Introduction}

Given the substantial amount of time people spend driving, there is an increasing demand for enjoyable in-car experiences that do not compromise safety. To make better use of their time, drivers tend to multi-task (e.g. checking traffic conditions, exchanging texts, etc.) by manipulating their In-Vehicle Infotainment (IVI) systems or mobile devices. Interacting with such devices while driving results in high cognitive load, which can amplify the driver's lack of focus and could lead to accidents.

While several IVI systems have been designed to keep the driving experience as safe as possible, it is still inconvenient for drivers to limit themselves to the systems that are part of the car. Many users would like to access their favorite applications everywhere, particularly inside their cars, despite the fact that some of these applications were not designed for a driving context. Many vehicles fail to provide a seamless transition for drivers as they enter and exit the vehicle since data usually does not flow into the car unless additional devices are brought in, and used while driving.

In addition to safety, social and emotional factors play a vital role in the driving experience, yet many IVI systems neglect these influences. For instance, car manufacturers do consider social factors of their driver demographic, like personality and 
emotion, as key aspects of car design [1]. Further, the driver's emotional state can have a significant impact on driver behavior and safety. Tired or stressed drivers are less likely to fully focus on the road, as compared to alert drivers. And angry, frustrated drivers are more prone to road rage, as compared to happy drivers.

These issues indicate a need for an IVI system that reinforces safety, manages information from the environment and the driver's personal device, and interacts with the driver in a social manner. Integrating these aspects into a unified architecture could make the driving experience safer, more efficient, and overall, more enjoyable.

\section{Related Work}

Tchankue, Wesson and Vogts addressed driver distraction while texting by presenting information upon arrival, and additional cues were included to express the priority level of the message [2]. These cues would allow the driver to decide when to deal with the received message. They found that these informative interruption cues were learned quickly and identified accurately to reduce cognitive load. However, this work does not explore the social aspect of driving and the possibility of taking proactive actions that reach out to other applications to offer more support to the driver.

Nass and Brave concluded that matching driver emotions with the IVI system's tone of voice improves overall in-car performance [3]. Matching happy drivers with an enthused voice and upset drivers with a subdued voice resulted in superior driving performance as opposed to matching opposite pairs. In [4], affective speech interfaces were explored as a means of seamless driver-vehicle communication. While these works reveal the benefits of speech-based interfaces, they do not explore information delivery in an expressive, sociable way to improve the quality of communication. If the IVI can express itself in a way that feels natural and familiar to the driver, then there could be a deeper understanding of the messages that it conveys.

Technologies like Bluetooth headsets and docking stations on windshields and dashboards allow drivers to access their mobile devices without compromising safety [5]. In [6], a system was designed that integrates the IVI system and the user's smartphone. Mobile devices run all phone services, while the IVI system is responsible for all input/output functions to handle a wider range of applications. While these systems are convenient, they degrade the user experience because they are highly reactive. The driver still has to initiate many actions to obtain necessary information.

\section{Core Contribution}

With motivations in mind from the previous sections, we have developed an Affective Intelligent Driving Agent (AIDA) [7], a socially expressive robot that acts as a friendly, in-car assistant, Fig. 1. AIDA uses the driver's mobile device as its face. Thus, AIDA uses app-based functionality to display facial expressions, manage and deliver task-specific information, and leverage aspects of the driver's daily life outside of the vehicle for deep personalization. 


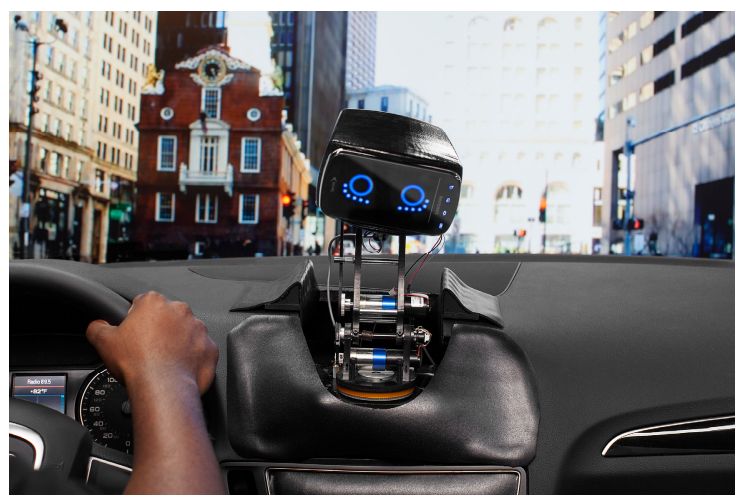

Fig. 1. In-car concept of the Affective Intelligent Driving Agent (AIDA)

AIDA allows users to accomplish their driving tasks while promoting in-car safety by keeping the phone out of the driver's hands. Since AIDA acts as an intermediary between the driver, the phone's applications, the vehicle and the environment, people will not lose access to the applications they need to perform their activities. Further, as opposed to traditional methods of driver-vehicle communication, e.g. flashing icons and chiming sounds, AIDA communicates with the driver through speech, coupled with expressive body movements.

\section{System Framework}

The robot consists of a five degree-of-freedom head and neck mechanism, which sits seamlessly in the dashboard when recessed, but conveys stronger expressions when extended. An Android smartphone acts as the face of the robot. The robot's plastic head shell was designed so that the driver can easily snap in the mobile device.

The Android phone is the core of AIDA's framework, which manages internal apps and sensor data, as well as communicates with the physical robot. We have developed an AIDA app, which encapsulates all of this functionality, displays facial expressions, and interconnects other apps. The AIDA app also queries external sources like the Internet for further assistance. The driver communicates with the device via speech commands and to a lesser extent, through tactile interactions.

In addition to the phone's standard apps, we have developed AIDA-specific apps (excluding the LTTS Susan app which was purchased through the Google Play Store) to assess AIDA's effectiveness as a proactive, social driving companion.

(a) LTTS Susan - A text-to-speech engine used to convert written text to verbal utterances using a female voice. This app is used to verbalize emoticons and text statements that the driver may receive during the driving task.

(b) Gas Station Finder (GSF) - Finds nearest gas stations given the driver's current location. Once the driver selects a station, the app navigates them appropriately.

(c) Travel Time Calculator (TTC) - Determines how long it will take for the driver 
to navigate to a certain location. The driver inputs an address and the app determines their travel time given the driver's current GPS coordinates.

(d) AIDA -This app integrates all context-specific apps into a seamless architect ture, while an expressive face acts as the main interface. The driver interacts with this app via speech and to a lesser extent, through touch.

(e) R1D1 - A communication hub between the phone and robot [8]. It sends motor positions to the robot given commands from the AIDA app.

\section{Experiment}

Our experiment examines peoples' attitudes towards AIDA and assesses user interactions with AIDA compared to a mobile device during mock driving tasks. For now, we are not assessing AIDA's impact on driver car handling (e.g. steering, response time, etc.). Instead, we are evaluating AIDA's ability to provide a more seamless way for the driver to handle the data flow between themselves, the car, and the outside world while promoting sociability.

\subsection{Protocol}

Upon arrival, participants were given an orientation, which explained the procedures of the experiment. We demonstrated how to use the smartphone including all necessary apps and services. Users were allowed to practice with the phone until they felt comfortable using it for the experiment. Users were then placed in an in-car environment where a laptop mounted atop a dashboard/steering wheel rig displayed a mock driving system. Users were videotaped while they performed the 15-minute task. Last, participants filled out a questionnaire regarding the interaction.

\subsection{Experimental Conditions}

Three conditions were used for the evaluation, Fig. 2. In Case PHONE, users completed the task with the smartphone alone, Fig. 2 (left). People used the Messaging, Calendar, Contacts, GSF, and TTC apps to assist them. This is similar to the way drivers currently use their smartphones in their vehicles. In Case AGENT, users completed the task with the AIDA app, while the phone was mounted on a stationary

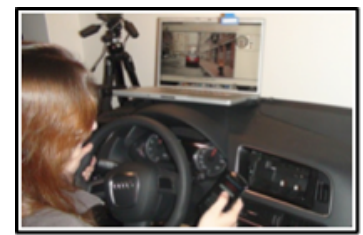

Case PHONE

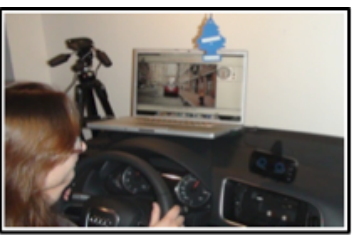

Case AGENT

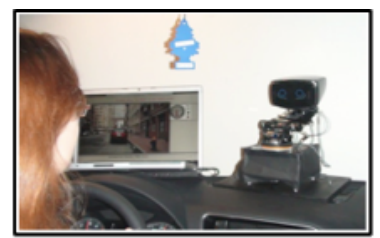

Case ROBOT

Fig. 2. Three experimental conditions 
dock, Fig. 2 (middle). This resembles existing in-car embedded screens and docking stations for driver assistance. In Case ROBOT, users completed the task with the AIDA app attached to the physical robot, Fig. 2 (right). To minimize distractions, the robot was programmed to move only when the car was stationary in the mock driving system. This explores an in-car interface that fuses driving assistance with sociability.

\subsection{Experimental Task}

Users had two events to attend while stress-inducing phone alerts and vehicle warnings occurred throughout the interaction. We encouraged users to act as naturally as possible and to perform any in-car actions (i.e., signaling, lane switching, accelerating, braking, etc.) that they would normally do while driving in the real world. Thus, users had the choice to make/answer phone calls or to send/read text messages. The R1D1 app handled communication between the driving system and phone/AIDA.

Two events were pre-programmed into the phone's calendar: 1) Lunch with the user's fictitious friend named Jaylen and 2) Salsa dancing lessons with another fictitious friend named Nancy. The locations and contact information associated with these events were also stored. The participant's goal was to arrive at these two events on time in the mock driving system. However, we crafted the system to force the user to become late for their first event due to traffic, irrespective of their driving performance. We also fashioned a low-gas vehicle warning when the user navigated to the second event. We also made phone calls and sent text messages to the user (pretending to be Nancy and Jaylen) at critical moments throughout the task. We monitored the interaction remotely in order to send these alerts at the appropriate times.

In PHONE, to navigate to the respective events, participants launched the Calendar app to find the appropriate event addresses. Once they knew the address, they then launched the TTC app to figure out their estimated travel time. We programmed the mock driving system to activate only once the user's travel time was established. If users chose to respond to our texts/calls throughout the interaction, they had to do so while trying to focus on the driving task. If users wanted to fill their gas tank in response to the low-gas warning, they launched the GSF app to assist them.

In AGENT, participants launched the AIDA app and AIDA proactively navigated the user to the event, which made beginning the task more seamless. Because the AIDA app encapsulates the Calendar and TTC apps, AIDA is aware of the driver's contacts and calendar events before they even enter the vehicle. Further, contextual awareness allows AIDA to better mitigate data flow between the driver and outside environment. For example, when the driving system notified the driver that they were late for their first event, AIDA preemptively asked the user if they wanted to send a text to Jaylen (i.e. us) notifying him of their tardiness. This way, users could communicate with the outside world while focusing on the driving task. Also, if users wanted to fill their tank in response to the low-gas warning, AIDA preemptively navigated them to the nearest station since the GSF app is integrated into the AIDA app.

In ROBOT, the interaction was similar, as previously described in AGENT. However, in addition to proactive assistance, the AIDA robot elicited direct 
non-verbal cues to the driver via body movements. Expressive body movements were coupled with AIDA's messages to the driver to promote deeper sociability.

\subsection{Hypotheses}

Taking insights from works in anthropomorphic interface technology, we predict three hypotheses. In [9], it was determined that affective interfaces had a major impact on reducing mental overload. Relating this work to our driving task, we predict:

H1 - Task Load: AGENT and ROBOT users will find the experimental task less mentally demanding and easier to complete than PHONE users.

In [10], people felt a stronger sense of cooperation and sociability with a robotic assistant versus an expressive agent. Thus, we predict:

H2 - Co-Supportive Teaming: AGENT and ROBOT users will see AIDA as an equal partner more than PHONE users with the smartphone. Further, this sentiment will be stronger felt in ROBOT than in AGENT.

H3 - Impact of Embodiment: ROBOT users will feel a deeper social bond with AIDA as a robot than AGENT users with AIDA as an on-screen agent only.

\subsection{Dependent Measures}

Hypotheses were evaluated through questionnaire responses and video footage. Survey questions from accepted sources provided us with standard methods to assess sociability and cooperation [11] and cognitive load [12]. Video data was used to assess user attention and mood. We coded for eye gaze, facial expressions, gestures, utterances and in-car actions. We define positive affect as smiles, laughs, excitement and positive utterances/gestures expressed by participants. Conversely, we define negative affect as frowns, confusion, anger, sadness and negative utterances/gestures.

\section{$6 \quad$ Results}

We recruited 44 participants (13 PHONE, 17 AGENT, and 14 ROBOT) from the \{location\} area. There were 20 males, 24 females and the mean age was 28.6 years.

Questionnaires were scored on a 5-point Likert Scale (1 = Strongly Disagree, 2 = Disagree, 3 = Neutral, 4 = Agree, $5=$ Strongly Agree). Mean $(M)$ and Standard Deviation $(\sigma)$ values of user responses were calculated for each experimental condition, Fig. 3. ANOVA $F$-values (AFV) were calculated to assess global variance. Pair-wise comparisons were then found using the Tukey-Kramer Method (TKM).

Video footage was transcribed by two objective individuals for behaviors discussed in Section 7. Krippendorff's alpha criterion was used to determine inter-coder reliability where $\alpha$-values between 0.8 and 1 (inclusive) suggest acceptable consistency. We found $\alpha=0.873$ for key behaviors highlighted in Table 1. Pair-wise comparisons between PHONE-AGENT $\left(\mathrm{T}_{\mathrm{PA}}\right)$, PHONE-ROBOT $\left(\mathrm{T}_{\mathrm{PR}}\right)$ and AGENT- ROBOT $\left(\mathrm{T}_{\mathrm{AR}}\right)$ were then found using TKM, Table 1. 


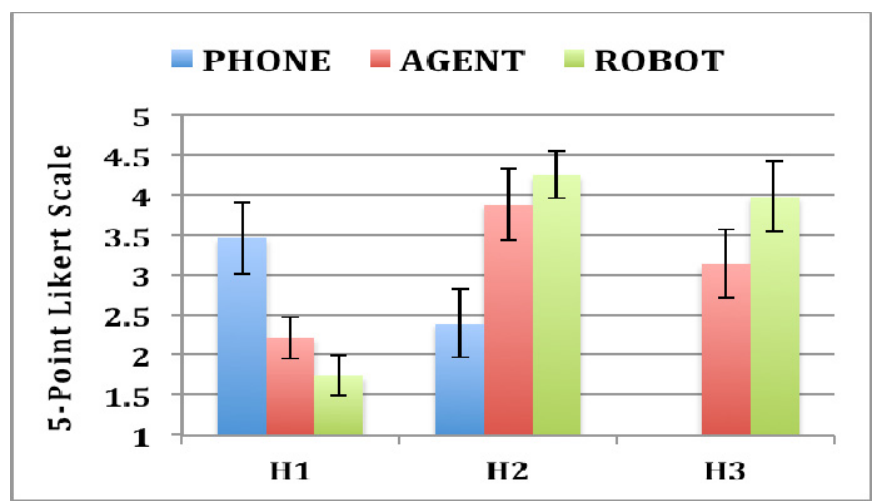

Fig. 3. Mean $(M)$ and Standard Deviation $(\sigma)$ of questionnaire responses (error $= \pm \sigma$ )

Table 1. Observations from video data with Tukey-Kramer Method (TKM) comparisons

\begin{tabular}{ccccccc}
\hline Behavior & PHONE & AGENT & ROBOT & $\mathrm{T}_{\mathrm{PA}}$ & $\mathrm{T}_{\mathrm{PR}}$ & $\mathrm{T}_{\mathrm{AR}}$ \\
\hline $\begin{array}{c}\text { a) Positive } \\
\text { Affect }\end{array}$ & 7.0 & 13.5 & 16.5 & 6.5 & 9.5 & 3.0 \\
$\begin{array}{c}\text { b) Negative } \\
\text { Affect }\end{array}$ & 5.5 & 2.0 & 1.5 & 3.5 & 4.0 & 0.5 \\
\hline
\end{tabular}

H1 - Fig. 3 (H1) shows the average replies to questions regarding high task load during the experiment. AFV revealed that most PHONE users felt that they experienced a high task load while AGENT and ROBOT users largely disagreed $(F(2,41)$ $=3.88, p=0.029)$. TKM comparisons showed significant differences between each pair-wise group $(\mathrm{T}(40,3)$, all $\mathrm{p}<0.05)$. Thus, ROBOT users felt less exhausted by the task than other users. Table 1 (b) illustrates that PHONE users expressed more negative affect throughout the task. TKM comparisons, T(40,3), showed significant differences between PHONE-AGENT and PHONE-ROBOT, but not AGENT-ROBOT.

H2 - Fig. 3 (H2) shows the mean responses to questions regarding the user's feeling that they were working as a team with the phone or AIDA to accomplish the driving task. PHONE users did not feel a sense of equal partnership with the phone as compared to AIDA users $(F(2,41)=10.26, p=0.0003)$. TKM comparisons, T $(40,3)$, showed significant differences between all pair-wise groups. Thus, users felt that the AIDA robot was more of a supportive teammate than the static AIDA display.

H3 - Table 1 (a) shows that AIDA users expressed more positive affect as compared to PHONE users $(F(2,41)=4.92, p=0.039)$. TKM comparisons, T $(40,3)$, revealed that ROBOT users expressed significantly more positive affect than AGENT users. Fig. 3 (H3) shows the mean responses to questions regarding the feeling that a mutual social bond existed between the user and AIDA. Users felt more mutual social awareness with the AIDA robot than the static display $(F(1,29)=5.89, p=0.003)$. 


\section{Discussion}

H1 - Task Load: Validated. Since AIDA users did not need to manipulate the device as much as PHONE users, they found the task less mentally demanding.

H2 - Co-Supportive Teaming: Validated. Due to its proactive support, AIDA was seen as an equal partner during the mock driving task more than the smartphone. This sentiment was stronger felt with the AIDA robot than the static agent.

H3 - Impact of Embodiment: Validated. The AIDA robot was able to elicit more personally directed non-verbal cues to the driver. Thus, users felt a deeper social bond with the embodied robot than the static display.

This work provides initial insights into AIDA's effectiveness at reducing task load and promoting sociability. Our results suggest that AIDA has the potential to better support drivers than smartphones alone during simulated driving tasks. They also suggest that the AIDA robot can promote sociability better than the static agent. Further investigation will explore AIDA's effectiveness during real-world driving tasks.

\section{References}

1. Bayley, M., Curtis, B., Lupton, K., Wright, C.C.: Vehicle Aesthetics and their Impact on the Pedestrian Environment. Technical report, Transportation Research D (2004)

2. Tchankue, P., Wesson, J., Vogts, D.: The Impact of an Adaptive User Interface on Reducing Driver Distraction. In: International Conference on Automotive User Interfaces, Salzburg, Austria (2011)

3. Nass, C., Brave, S.: Wired for Speech: How Voice Activates and Advances the HumanComputer Relationship. MIT Press, Michigan (2005)

4. McAllister, G., McKenna, S.J., Ricketts, I.W.: Towards a Non-Contact Driver-Vehicle Interface. In: Intelligent Transportation Systems (2000)

5. Bühler, D., Vignier, S., Heisterkamp, P., Minker, W.: Safety and Operating Issues for Mobile Human-Machine Interfaces. In: International Conference on Intelligent User Interfaces, Miami, Florida (2003)

6. Bose, R., Brakensiek, J., Park, K.Y.: Terminal Mode - Transforming mobile devices into automotive application platforms. In: International Conference on Automotive User Interfaces, Pittsburg, Pennsylvania (2010)

7. Foen, N.: Exploring the Human-Car Bond through an Affective Intelligent Driving Agent. Master's thesis. MIT, Cambridge, MA (2012)

8. Isla, D., Burke, R., Downie, M., Blumberg, B.: A layered brain architecture for synthetic characters. In: The International Joint Conference of Artificial Intelligence (2001)

9. Nass, C., Steuer, J., Tauber, E.R.: Computers are social actors. In: Special Interest Group on Computer Human Interaction: Human Factors in Computing (1994)

10. Kidd, C., Brezeal, C.: Comparison of social presence in robots and animated characters. Interaction Journal Studies (2005)

11. Takayama, L., Groom, V., Nass, C.: I'm sorry, Dave: I'm afraid I won't do that: social aspects of human-agent conflict. In: Human Factors in Computing Systems. ACM, New York (2009)

12. Sweller, J.: Cognitive load during problem solving: Effects on learning. In: Cognitive Science, vol. 257 (1988) 